\title{
Distributed bounded-error state estimation
}

\author{
Michel Kieffer \\ L2S - CNRS - SUPELEC - Univ Paris-Sud, \\ 3 rue Joliot-Curie, 91192 Gif-sur-Yvette, France \\ (Tel: $0033(0) 1698517$ 32; e-mail: kieffer@lss.supelec.fr).
}

\begin{abstract}
This paper presents a distributed bounded-error state estimation algorithm suited, e.g., to measurement processing by a network of sensors. Contrary to centralized estimation, where all data are collected to a central processing unit, here, each data is processed locally by the sensor, the results are broadcasted to the network and taken into account by the other sensors. A first analysis of the conditions under which distributed and centralized estimators provide the same results is presented. An application to the tracking of a moving source using a network of sensors measuring the strength of the signal emitted by the source is considered *.

* This work has been partly supported by NEWCOM++
\end{abstract}

\section{INTRODUCTION}

State estimation usually involves a state equation describing the evolution of the considered system and measurements provided by sensors Gelb [1974]. In a classical centralized estimation scheme, all measurements are gathered at a central processing unit (CPU), responsible for all the signal processing. Alternatively, computations may be performed in a decentralized, or distributed, manner. In such approach, each sensor processes its own measurements together with the data received from other sensors Speyer [1979], Durrant-White et al. [1990], Rao et al. [1993]. The main advantage of the latter approach is its robustness to failures of sensors or of the CPU: in the distributed context, even if one or more sensors fail, other sensors continue to provide state estimates. Distributed estimation techniques are currently gaining a growing interest, thanks to the availability of nodes in wireless sensor networks with increased battery and processing power Kay and Mattern [2004], Haenselmann [2006].

Distributed state estimation is more complex than centralized estimation: the limited computational complexity and transmission capabilities have to be taken into account. This raises several problem, among which one may cite the choice of the type of data to transmit between sensors, of the transmission protocol, of the way these data have to be processed by the sensors, etc.

For what concerns the type of data, measurements or estimates may be transmitted. When the number of node in the network is larger than the dimension of the state vector, transmitting measurements to all sensors may result in a high communication load, except if data are very coarsely quantized, as done in Ribeiro et al. [2006]. Thus, estimates are usually transmitted from one sensor to the others, as in Rao et al. [1993], López-Orozco et al. [2000], Olfati-Saber [2005], Alriksson and Rantzer [2007], where decentralized version of the Kalman filter Kalman [1960] were proposed. As for centralized Kalman filtering, state perturbations and measurement noise are assumed to be described by Gaussian probability density functions. Moreover, the dy- namical part of the state equation is usually assumed to be linear or is linearized. As a consequence, the best than can be achieved is a an estimate and its associated confidence region: no guarantee may be provided as regards quality of the obtained results.

In the approach proposed in this paper, state perturbation and measurement noise will be assumed to be bounded with known bounds. The aim of distributed state estimation in such bounded-error context is to evaluate, at any node of the network, a set containing all state values which are consistent with the information (model, noise bounds, measurements, data of neighboring sensors) available at that node. For that purpose, set estimates will be exchanged between nodes of the network. The resulting set estimator is guaranteed, since each set evaluated by the nodes contains the actual state value, as in centralized bounded-error state estimation Kieffer et al. [1998, 2002], provided that the hypotheses on the model and noise bounds are satisfied.

The paper is organized as follows. Section 2 presents the model, before recalling the principle of idealized centralized bounded-error state estimation in Section 3. Its distributed extension is provided in Section 4. The conditions under which the distributed and the centralized algorithms perform similarly is provided. Using interval analysis Moore [1966], Jaulin et al. [2001], it will be possible to build an implementable counterpart of the idealized distributed state estimation algorithm. Finally, Section 5 presents the application of the preceding algorithm to the tracking of a source emitting waves which amplitude is measured by the nodes of a wireless sensor network.

\section{BOUNDED-ERROR DISCRETE-TIME STATE ESTIMATION}

Consider a system described by a model consisting of a discrete-time state equation

$$
\mathbf{x}_{k}=\mathbf{f}_{k}\left(\mathbf{x}_{k-1}, \mathbf{w}_{k}, \mathbf{u}_{k}\right)
$$

where $\mathbf{x}_{k}$ is the state vector of the model at time instant $k$ (the sampling period is $T$ ). The state perturbation vector 
$\mathbf{w}_{k}$ accounts for unmodeled parts of the system and is assumed to remain in some known box $[\mathbf{w}]$. The input vector $\mathbf{u}_{k}$ is also assumed known. At $k=0, \mathbf{x}_{0}$ is only assumed to belong to some (possibly large) known set $\mathbb{X}_{0}$.

Assume that at time $k$, each node $\ell=1 \ldots L$ of a sensor network has access to a noisy measurement vector $\mathbf{y}_{k}^{\ell}$. The measurement process is described by the observation equations

$$
\mathbf{y}_{k}^{\ell}=\mathbf{g}_{k}^{\ell}\left(\mathbf{x}_{k}, \mathbf{v}_{k}^{\ell}\right), \ell=1 \ldots L
$$

where $\mathbf{v}_{k}^{\ell}$ is the measurement noise, assumed bounded in some known box $[\mathbf{v}]$. Usual observation equations are

$$
\mathbf{g}_{k}^{\ell}\left(\mathbf{x}_{k}, \mathbf{v}_{k}^{\ell}\right)=\mathbf{h}_{k}^{\ell}\left(\mathbf{x}_{k}\right)+\mathbf{v}_{k}^{\ell}
$$

or

$$
\mathbf{g}_{k}^{\ell}\left(\mathbf{x}_{k}, v_{k}^{\ell}\right)=\mathbf{h}_{k}^{\ell}\left(\mathbf{x}_{k}\right) \cdot v_{k}^{\ell}
$$

depending on whether the measurement noise is additive or multiplicative.

Centralized and distributed state estimation aims at evaluating at each time instant $k$ the set of all state values that are consistent with the models (1) and (2), the measurements, and the bounds on the state perturbation and measurement noise. The main difference between centralized and distributed estimation is that in the former, all measurements are available at a given point, whereas in the latter, estimation is performed at each node, and data has to be exchanged between sensors.

\section{CENTRALIZED DISCRETE-TIME STATE ESTIMATION IN A BOUNDED-ERROR CONTEXT}

This section briefly summarizes centralized state estimation, since it constitutes the reference which distributed algorithms should reach.

When all measurements at time $k$ are available at a CPU, one gets from (1) and (2)

$$
\left\{\begin{array}{l}
\mathbf{x}_{k}=\mathbf{f}_{k}\left(\mathbf{x}_{k-1}, \mathbf{w}_{k}, \mathbf{u}_{k}\right), \\
\mathbf{y}_{k}=\mathbf{g}_{k}\left(\mathbf{x}_{k}, \mathbf{v}_{k}\right)
\end{array}\right.
$$

with $\mathbf{y}_{k}^{\mathrm{T}}=\left(\left(\mathbf{y}_{k}^{1}\right)^{\mathrm{T}} \ldots\left(\mathbf{y}_{k}^{L}\right)^{\mathrm{T}}\right)$ and $\mathbf{v}_{k}^{\mathrm{T}}=\left(\left(\mathbf{v}_{k}^{1}\right)^{\mathrm{T}} \ldots\left(\mathbf{v}_{k}^{L}\right)^{\mathrm{T}}\right)$. Summarizing the information available at time $k$, one obtains

$$
\mathcal{I}_{k}=\left\{\mathbb{X}_{0},\left\{\left[\mathbf{w}_{j}\right]\right\}_{j=1}^{k},\left\{\left[\mathbf{v}_{j}\right]\right\}_{j=1}^{k},\left\{\left[\mathbf{y}_{j}\right]\right\}_{j=1}^{k}\right\}
$$

Performing a centralized bounded-error state estimation at time $k$ aims at characterizing the set $\mathbb{X}_{k \mid k}$ of all values of $\mathbf{x}_{k}$ that are consistent with (1), (2), and $\mathcal{I}_{k}$. One may propose an idealized algorithm Kieffer et al. [2002], alternating, as the Kalman filter a prediction step involving (1)

$$
\mathbb{X}_{k \mid k-1}=\left\{\mathbf{f}_{k}\left(\mathbf{x}, \mathbf{w}, \mathbf{u}_{k}\right) \mid \mathbf{x} \in \mathbb{X}_{k-1 \mid k-1}, \mathbf{w} \in[\mathbf{w}]\right\}
$$

and a correction step accounting for the new measurement using (2)

$$
\mathbb{X}_{k \mid k}=\left\{\mathbf{x} \in \mathbb{X}_{k \mid k-1} \mid \mathbf{y}_{k}=\mathbf{g}_{k}(\mathbf{x}, \mathbf{v}), \mathbf{v} \in[\mathbf{v}]^{L}\right\} .
$$

The two steps of the idealized algorithm are depicted in Figure 1.

This idealized algorithm requires the evaluation of the direct image of a set by a function in the prediction step (7) and the evaluation of the inverse image in the correction step (8). To get an implementable counterpart of this algorithm, first one has to wrap the sets $\mathbb{X}_{k \mid k-1}$ and

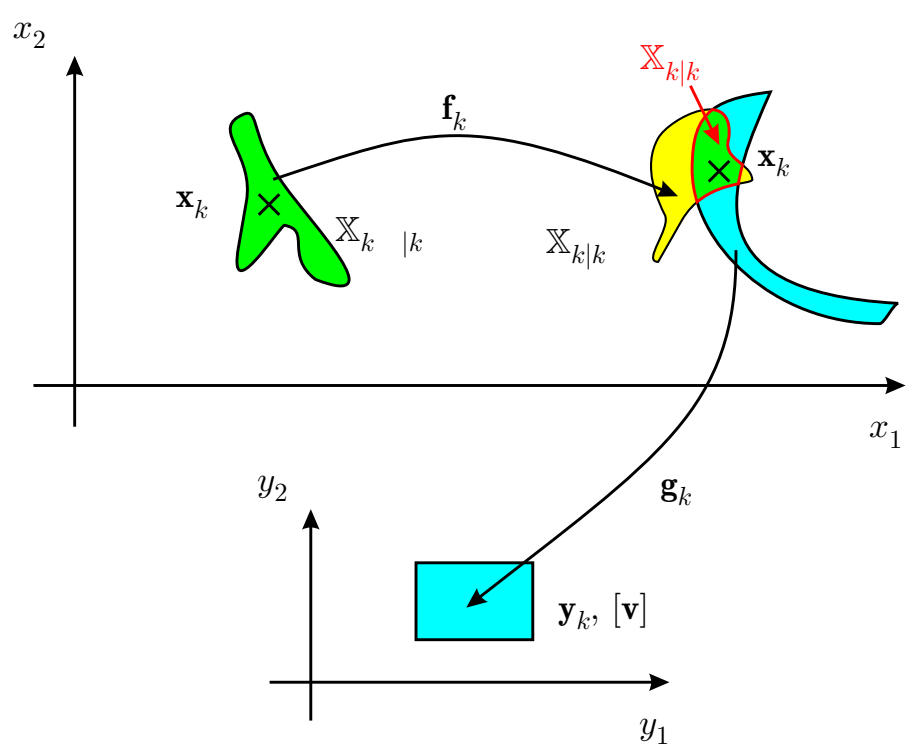

Fig. 1. Idealized recursive bounded-error state estimator $\mathbb{X}_{k \mid k}$, which can take any shape, by sets $\overline{\mathbb{X}}_{k \mid k-1}$ and $\overline{\mathbb{X}}_{k \mid k}$ allowing a parametric description. Candidate wrappers are for example ellipsoids Schweppe [1973], zonotopes Alamo et al. [2003], interval vectors or boxes Moore [1979] or union of boxes Kieffer et al. [2002, 2001]. Due to the wrapping effect, some $\mathbf{x} \in \overline{\mathbb{X}}_{k \mid k}$ may not correspond to values of the state vector consistent with (6). Depending on the wrapper, prediction and correction steps may require further hypotheses on the state equation. When ellipsoids and zonotopes are considered, (1) and (2) have to be linear Schweppe [1973], Alamo et al. [2003]. This condition is not required when considering boxes or unions of boxes, thanks to interval analysis, which provides tools to evaluate outer approximations of (7) and (8), see Kieffer et al. [2002], Jaulin et al. [2001].

\section{DISTRIBUTED STATE ESTIMATION}

When considering distributed state estimation, all measurements are not directly available at each node of the network. The set estimate $\mathbb{X}_{k \mid k}^{\ell}$ provided by the $\ell$-th node is thus such that $\mathbb{X}_{k \mid k}^{\ell} \subset \mathbb{X}_{k \mid k}$. Ideally, the data exchange between sensors should allow to get

$$
\mathbb{X}_{k \mid k}^{\ell}=\mathbb{X}_{k \mid k}, \ell=1 \ldots L
$$

To establish conditions under which (9) is satisfied, some notions of graph theory have to be recalled. For more details, the reader is referred to Harary [1994], Bollobás [1998].

\subsection{Description of the network}

The network of $L$ sensors is represented by a graph $\mathcal{G}=$ $(\mathcal{V}, \mathcal{E}) . \mathcal{V}$ is the set of $L$ vertices of the graph, each vertex representing a node of the network and $\mathcal{E}$ is the set of edges of the graph. An edge $\{k, \ell\} \in \mathcal{E}$ connecting two vertices $k \in \mathcal{V}$ and $\ell \in \mathcal{V}$ indicates that the two corresponding nodes are able to directly exchange information; the graph is thus undirected. In what follows, it is assumed that $\mathcal{G}$ is entirely connected, i.e., that there is always a path from 
any vertex to any other vertex in $\mathcal{G}$ and that each vertex is connected to itself.

The distance between two vertices in $\mathcal{G}$ is the number of edges in a shortest path connecting them. Consider a vertex $\ell \in \mathcal{V}$, then

$$
\mathcal{C}(\{\ell\})=\{k \in \mathcal{V} \mid(k, \ell) \in \mathcal{E}\}
$$

denotes the set of all vertices that are directly connected to $\ell$, i.e., that are at a distance not larger than one of $\ell$. More generally, for any $\mathcal{W} \subset \mathcal{V}, \mathcal{C}(\mathcal{W}) \subset \mathcal{V}$ is the set of all vertices which are at a distance not larger than one from a given vertex of $\mathcal{W}$. The set

$$
\mathcal{C}(\mathcal{C}(\{\ell\}))=\mathcal{C}^{2}(\{\ell\})
$$

contains thus all vertices that are at a distance not larger than two of $\ell$. More generally, $\mathcal{C}^{n}(\{\ell\})$ contains all vertices that are at a distance not larger than $n$ of $\ell$. The eccentricity $\varepsilon$ of a vertex $\ell \in \mathcal{V}$ is the largest distance between $\ell$ and any other vertex in $\mathcal{G}$. Finally, the diameter $d$ of $\mathcal{G}$ is the maximum eccentricity of any vertex in $\mathcal{G}$.

\subsection{Hypotheses and idealized algorithm.}

The following measurement processing and communication protocol will be considered.

At time $k$, each sensor processes its own measurement $\mathbf{y}_{k}^{\ell}$, to get $\mathbb{X}_{k \mid k}^{\ell, 0}$. Between time $k$ and $k+1$, a first round trip is considered $(r=1)$ during which each sensor $\ell$ broadcasts its own estimate $\mathbb{X}_{k \mid k}^{\ell, r-1}$ to all the sensors of the network (only those which are directly connected to $\ell$ receive the information). Each sensor may communicate in turn using simple time division multiple access, or simultaneously using, e.g., code division multiple access Sklar [2001]. Then each sensor $\ell$ receives and processes $\mathbb{X}_{k \mid k}^{s, r-1}, s \in \mathcal{C}(\{\ell\})$ to get $\mathbb{X}_{k \mid k}^{s, r}$. Depending on the sampling time $T$, more round trips $(r>1)$ may be considered. Just before time $k+1$, each sensor $\ell$ builds a final estimate $\mathbb{X}_{k \mid k}^{\ell}$.

This way of processing and transmitting information leads to the following idealized distributed algorithm.

For each sensor $\ell=1 \ldots L$,

(1) At time $k$

$$
\begin{aligned}
\mathbb{X}_{k \mid k-1}^{\ell} & =\left\{\mathbf{f}_{k}\left(\mathbf{x}, \mathbf{w}, \mathbf{u}_{k}\right) \mid \mathbf{x} \in \mathbb{X}_{k-1 \mid k-1}^{\ell}, \mathbf{w} \in[\mathbf{w}]\right\} \\
\mathbb{X}_{k \mid k}^{\ell, 0} & =\left\{\mathbf{x} \in \mathbb{X}_{k \mid k-1}^{\ell} \mid \mathbf{y}_{k}^{\ell}=\mathbf{g}_{k}^{\ell}(\mathbf{x}, \mathbf{v}), \mathbf{v} \in[\mathbf{v}]\right\}
\end{aligned}
$$

(2) Between $k$ and $k+1$, for $r=1$ to $R_{\max }$ (number of round trips)

$$
\mathbb{X}_{k \mid k}^{\ell, r}=\bigcap_{s \in \mathcal{C}(\{\ell\})} \mathbb{X}_{k \mid k}^{s, r-1}
$$

(3) Just before $k+1$

$$
\mathbb{X}_{k \mid k}^{\ell}=\mathbb{X}_{k \mid k}^{\ell, R_{\max }}
$$

As for the centralized algorithm, the idealized distributed algorithm requires the evaluation of the direct and inverse images of a set by a function. Proposition 1 gives some conditions under which the distributed approach gives results similar to the centralized one.
Proposition 1. Consider a WSN of $L$ nodes represented by an entirely connected graph $\mathcal{G}=(\mathcal{V}, \mathcal{E})$ of diameter $d$. Assume that at time $k-1, \mathbb{X}_{k-1 \mid k-1}^{\ell}=\mathbb{X}_{k-1 \mid k-1}$ for all $\ell \in \mathcal{V}$. If the number of roundtrips $R_{\max }$ satisfies $R_{\max } \geqslant d$, then one has at time $k$

$$
\mathbb{X}_{k \mid k}^{\ell}=\mathbb{X}_{k \mid k}
$$

for all $\ell \in \mathcal{V}$.

Proof. Consider a vertex $\ell \in \mathcal{V}$. Since $\mathbb{X}_{k-1 \mid k-1}^{\ell}=$ $\mathbb{X}_{k-1 \mid k-1}$, after the prediction step (12), $\mathbb{X}_{k \mid k-1}^{\ell}=\mathbb{X}_{k \mid k-1}$, where $\mathbb{X}_{k \mid k-1}$ is provided by (7). The first correction step done at $\ell$ involves only the measurement vector $\mathbf{y}_{k}^{\ell}$ to get $\mathbb{X}_{k \mid k}^{\ell, 0}$. After the first roundtrip, the estimate at $\ell$ becomes

$$
\begin{aligned}
\mathbb{X}_{k \mid k}^{\ell, 1} & =\bigcap_{s \in \mathcal{C}(\{\ell\})} \mathbb{X}_{k \mid k}^{s, 0} \\
& =\bigcap_{s \in \mathcal{C}(\{\ell\})}\left\{\mathbf{x} \in \mathbb{X}_{k \mid k-1}^{s} \mid \mathbf{y}_{k}^{s}=\mathbf{g}_{k}^{s}(\mathbf{x}, \mathbf{v}), \mathbf{v} \in[\mathbf{v}]\right\} \\
& =\bigcap_{s \in \mathcal{C}(\{\ell\})}\left\{\mathbf{x} \in \mathbb{X}_{k \mid k-1} \mid \mathbf{y}_{k}^{s}=\mathbf{g}_{k}^{s}(\mathbf{x}, \mathbf{v}), \mathbf{v} \in[\mathbf{v}]\right\} \\
& =\left\{\mathbf{x} \in \mathbb{X}_{k \mid k-1} \mid \mathbf{y}_{k}^{\mathcal{C}(\{\ell\})}=\mathbf{g}_{k}^{\mathcal{C}(\{\ell\})}(\mathbf{x}, \mathbf{v}), \mathbf{v} \in[\mathbf{v}]\right\},
\end{aligned}
$$

where $\mathbf{y}_{k}^{\mathcal{C}(\{\ell\})}$ and $\mathbf{g}_{k}^{\mathcal{C}(\{\ell\})}(\mathbf{x}, \mathbf{v})$ are the vector and function consisting of the concatenation of all $\mathbf{y}_{k}^{s}$ and $\mathbf{g}_{k}^{s}(\mathbf{x}, \mathbf{v})$, with $s \in \mathcal{C}(\{\ell\})$.

After a second roundtrip, the estimate at $\ell$ becomes

$$
\begin{aligned}
\mathbb{X}_{k \mid k}^{\ell, 2} & =\bigcap_{s \in \mathcal{C}(\{\ell\})} \mathbb{X}_{k \mid k}^{s, 1} \\
& =\bigcap_{s \in \mathcal{C}(\{\ell\})}\left\{\mathbf{x} \in \mathbb{X}_{k \mid k-1} \mid \mathbf{y}_{k}^{\mathcal{C}(\{s\})}=\mathbf{g}_{k}^{\mathcal{C}(\{s\})}(\mathbf{x}, \mathbf{v}), \mathbf{v} \in[\mathbf{v}]\right\} \\
& =\left\{\mathbf{x} \in \mathbb{X}_{k \mid k-1} \mid \mathbf{y}_{k}^{\mathcal{C}(\mathcal{C}(\{\ell\}))}=\mathbf{g}_{k}^{\mathcal{C}(\mathcal{C}(\{\ell\}))}(\mathbf{x}, \mathbf{v}), \mathbf{v} \in[\mathbf{v}]\right\} \\
& =\left\{\mathbf{x} \in \mathbb{X}_{k \mid k-1} \mid \mathbf{y}_{k}^{\mathcal{C}^{2}(\{\ell\})}=\mathbf{g}_{k}^{\mathcal{C}^{2}(\{\ell\})}(\mathbf{x}, \mathbf{v}), \mathbf{v} \in[\mathbf{v}]\right\}
\end{aligned}
$$

Similarly, after $R_{\max }$ roundtrips, one gets at $\ell$ $\mathbb{X}_{k \mid k}^{\ell, R_{\max }}=\left\{\mathbf{x} \in \mathbb{X}_{k \mid k-1} \mid \mathbf{y}_{k}^{\mathcal{C}^{R_{\max }}(\{\ell\})}=\mathbf{g}_{k}^{\mathcal{C}^{R_{\max }}(\{\ell\})}(\mathbf{x}, \mathbf{v}), \mathbf{v} \in[\mathbf{v}]\right\}$

It is now enough to show that $\mathcal{C}^{R_{\max }}(\{\ell\})=\mathcal{V}$ in order to prove that $\mathbb{X}_{k \mid k}^{\ell, R_{\max }}=\mathbb{X}_{k \mid k}$. First, one has $\mathcal{C}^{R_{\max }}(\{\ell\}) \subset \mathcal{V}$. Assume now that there exists some $k \in \mathcal{V}$ such that $k \notin \mathcal{C}^{R_{\max }}(\{\ell\})$. This means that $k$ lies at a distance strictly larger than $R_{\max }$ from $\ell$. Since the diameter $d$ of $\mathcal{G}$ is lower than $R_{\max }$ the distance between two vertices is necessarily lower than $d$, which contradicts the initial assumption. Thus any $k \in \mathcal{V}$ satisfies $k \in \mathcal{C}^{R_{\max }}(\{\ell\})$ and $\mathcal{C}^{R_{\max }}(\{\ell\})=\mathcal{V}$.

The result of Proposition 1 mainly shows that when there are enough information exchanges between sensors, the distributed estimate converges at any sensor to the centralized estimate. What is more interesting is that the number of roundtrips needed for convergence depends only on the diameter of the graph associated with the WSN.

When $R_{\max }<d$, the situation is much more complex, since not all sensors will have access to all measurements (or to their contribution to the estimation of $\mathbf{x}_{k}$ ). For the first 
roundtrips at time $k+1$, sensor $\ell$ will have to broadcast information about $\mathbb{X}_{k+1 \mid k+1}^{\ell, r}$, but also about $\mathbb{X}_{k \mid k}^{\ell, r}$, as long as $\mathbb{X}_{k \mid k}^{\ell, r}$ has not converged to $\mathbb{X}_{k \mid k}$. Again, the diameter of the graph plays a crucial role. Further analysis is still required.

\subsection{Practical algorithm}

The implementation of the distributed algorithm requires the same wrappers as for the centralized algorithm. The complexity of the wrapper impacts the amount of information that has to be transmitted to the other nodes.

In this paper, a basic version of the algorithm is considered, where sets are wrapped by boxes. This simple description allows to perform the prediction step using basic tools from interval analysis, such as interval evaluations for the prediction step. The correction step is implemented via interval constraint propagation Jaulin et al. [2001]. The advantage of dealing with boxes is that interval analysis may readily be implemented on chips with reduced computational capabilities, see Piskorski et al. [2006]. A more sophisticated version could involve description of sets using subpavings, a prediction step implemented using IMAGESP Kieffer et al. [2002] and SIVIA Jaulin and Walter [1993] combined with interval constraint propagation for the correction step.

\section{APPLICATIONS}

For the application part, a problem of source tracking from readings of signal strength (RSS) data. Centralized approaches have been proposed to solve this problem for acoustic sources Sheng and $\mathrm{Hu}$ [2005] and for sources emitting electromagnetic waves, see, e.g., Kontkanen et al. [2004], Gustafsson and Gunnarsson [2005], Gezici et al. [2005]. In the first case, some knowledge of the decay rate of the RSS (path loss exponent) is needed for efficient nonlinear least squares estimation. In the second case, an off-line training phase is required to allow maximum a posteriori localization. In both cases, a good initial guess of the location of the source facilitates convergence to the global minimum of the cost function. Distributed approaches have also been employed, e.g., in Rabbat and Nowak [2004], where a distributed version of a nonlinear least squares solver has been presented. When badly initialized, it suffers from the same convergence problems as the centralized approach, as illustrated in Hero III and Blatt [2005], which advocates projection on convex sets. Nevertheless, the latter requires an accurate knowledge of the source signal strength and of the path loss exponent.

\subsection{Models}

The sensors are assumed to be static, and their location is known and denoted by $\mathbf{r}^{\ell} \in \mathbb{R}^{2}, \ell=1 \ldots L$. The state of the source at time instant $k$ is $\mathbf{x}_{k}=\left(\theta_{k}^{1}, \theta_{k}^{2}, \varphi_{k}^{1}, \varphi_{k}^{2}\right)^{\mathrm{T}} \in \mathbb{R}^{4}$, where $\boldsymbol{\theta}_{k}=\left(\theta_{k}^{1}, \theta_{k}^{2}\right)^{\mathrm{T}}$ represents the location of the source and $\varphi_{k}=\left(\varphi_{k}^{1}, \varphi_{k}^{2}\right)^{\mathrm{T}}$ its speed.

The evolution of the source is assumed to be described by

$$
\mathbf{x}_{k}=\left(\begin{array}{cccc}
1 & 0 & T & 0 \\
0 & 1 & 0 & T \\
0 & 0 & 1 & 0 \\
0 & 0 & 0 & 1
\end{array}\right) \mathbf{x}_{k-1}+T\left(\begin{array}{c}
0 \\
0 \\
w_{1} \\
w_{2}
\end{array}\right)
$$

Since the inputs are unknown, they are considered as bounded state perturbations, thus, $w_{1} \in[w]$ and $w_{2} \in[w]$.

The mean power $\bar{P}_{\mathrm{dB}}\left(d_{k}^{\ell}\right)$ (in $\mathrm{dBm}$ ) received by the $\ell$-th sensor is described by

$$
\bar{P}_{\mathrm{dB}}\left(d_{k}^{\ell}\right)=P_{0}-10 n_{\mathrm{p}} \log \frac{d_{k}^{\ell}}{d_{0}}
$$

where $P_{0}$ is the power received at a reference distance $d_{0}, n_{\mathrm{p}}$ is the path-loss exponent, and $d_{k}^{\ell}=\left|\mathbf{r}^{\ell}-\boldsymbol{\theta}_{k}\right|$, see Okumura et al. [1968]. The received power is assumed to lie within some bounds

$$
P_{\mathrm{dB}}(d) \in\left[P_{0}-10 n_{\mathrm{p}} \log \frac{d}{d_{0}}-e, P_{0}-10 n_{\mathrm{p}} \log \frac{d}{d_{0}}+e\right],
$$

where $e$ is assumed known.

Assuming that $P_{0}, d_{0}$, and $n_{\mathrm{p}}$ are known, the RSS by sensor $\ell=1 \ldots L$ may be rewritten as

$$
y_{k}^{\ell}\left(\boldsymbol{\theta}_{k}, v_{\ell}\right)=h_{\ell}\left(\boldsymbol{\theta}_{k}\right) v_{\ell}
$$

with

$$
h_{\ell}\left(\boldsymbol{\theta}_{k}\right)=\frac{A}{\left|\mathbf{r}^{\ell}-\boldsymbol{\theta}_{k}\right|^{n_{\mathrm{p}}}}, A=10^{P_{0} / 10} d_{0}^{n_{\mathrm{p}}}
$$

and $v_{\ell} \in[v]=\left[10^{-e / 10}, 10^{e / 10}\right]$. The noise, additive in the $\log$ domain is multiplicative in the normal domain.

\subsection{Prediction step: direct image evaluation}

Assume that the estimate at time $k-1$ for node $\ell$ is $\left[\mathbf{x}_{k-1 \mid k-1}^{\ell}\right]$. The estimate $\left[\mathbf{x}_{k \mid k-1}^{\ell}\right]$ may be obtained from (17) as follows

$$
\left[\mathbf{x}_{k \mid k-1}^{\ell}\right]=\left(\begin{array}{cccc}
1 & 0 & T & 0 \\
0 & 1 & 0 & T \\
0 & 0 & 1 & 0 \\
0 & 0 & 0 & 1
\end{array}\right)\left[\mathbf{x}_{k-1 \mid k-1}^{\ell}\right]+T\left(\begin{array}{c}
0 \\
0 \\
{[w]} \\
{[w]}
\end{array}\right)
$$

\subsection{Correction step: interval constraint propagation}

At sensor $\ell, y^{\ell} \in\left[y^{\ell}\right]$ is measured. Some boxes $\left[\mathbf{x}_{k}^{\ell}\right]$ is assumed to be available from the prediction step, or as results transmitted by the other sensors to sensor $\ell$. The parameter vector has to satisfy the constraint provided by the RSS model

$$
y^{\ell}-\frac{A}{\left|\mathbf{r}^{\ell}-\boldsymbol{\theta}_{k}^{\ell}\right|^{n_{\mathrm{p}}}}=0 .
$$

Using interval constraint propagation, it is possible to reduce the domain for the variables using (22). The contracted domains may be written as

$$
\left\{\begin{array}{l}
{\left[y^{\ell}\right]^{\prime}=\left[y^{\ell}\right] \cap \frac{A}{\left|\mathbf{r}^{\ell}-\left[\boldsymbol{\theta}_{k}^{\ell}\right]\right|^{n_{\mathrm{p}}}},} \\
{\left[\theta_{k, 1}^{\ell}\right]^{\prime}=\left[\theta_{k, 1}^{\ell}\right] \cap\left(r_{1}^{\ell} \pm \sqrt{\left.\left(A /\left[y^{\ell}\right]^{\prime}\right)^{2 / n_{\mathrm{p}}-\left(r_{2}^{\ell}-\left[\theta_{k, 2}^{\ell}\right]\right)^{2}}\right)}\right.} \\
{\left[\theta_{k, 2}^{\ell}\right]^{\prime}=\left[\theta_{k, 2}^{\ell}\right] \cap\left(r_{2}^{\ell} \pm \sqrt{\left.\left(A /\left[y^{\ell}\right]^{\prime}\right)^{2 / n_{\mathrm{p}}-\left(r_{1}^{\ell}-\left[\theta_{k, 1}^{\ell}\right]\right)^{2}}\right)} .\right.}
\end{array}\right.
$$




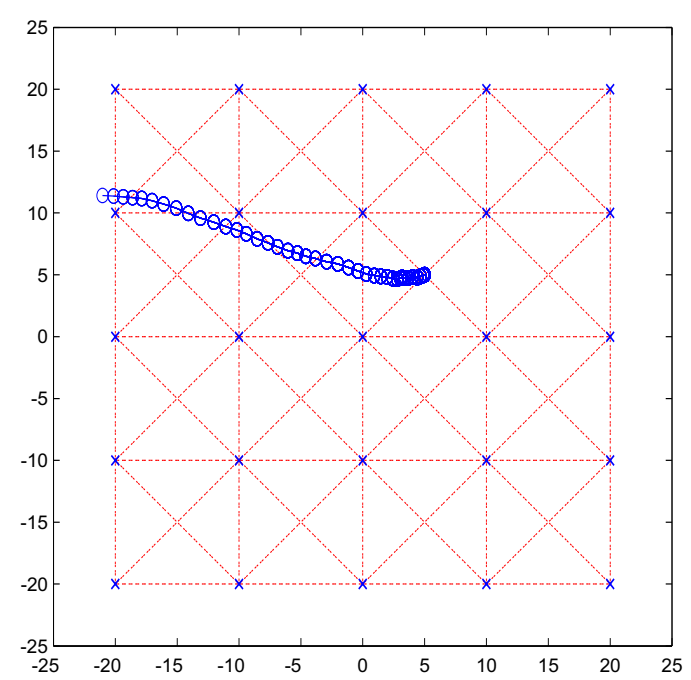

Fig. 2. Trajectory of the source (o); each sensor is represented by a cross $(\mathrm{x})$, distances are in meters

Once the estimate for $\boldsymbol{\theta}_{k}^{\ell}$ has been improved, it may be useful to use (21) to improve the estimation of the speed of the source as follows

$$
\left\{\begin{array}{l}
{\left[\varphi_{k, 1}^{\ell}\right]^{\prime}=\left[\varphi_{k, 1}^{\ell}\right] \cap\left(\frac{\left[\theta_{k, 1}^{\ell}\right]^{\prime}-\left[\theta_{k-1,1}^{\ell}\right]}{T}+T[w]\right),} \\
{\left[\varphi_{k, 2}^{\ell}\right]^{\prime}=\left[\varphi_{k, 2}^{\ell}\right] \cap\left(\frac{\left[\theta_{k, 2}^{\ell}\right]^{\prime}-\left[\theta_{k-1,2}^{\ell}\right]}{T}+T[w]\right) .}
\end{array}\right.
$$

Each sensor will perform this constraint propagation before transmitting its updated estimate to its neighbours.

\section{RESULTS}

A field of $50 \mathrm{~m} \times 50 \mathrm{~m}$ is considered, with its origin at the center. A WSN of $L=25$ sensors with communication range of $15 \mathrm{~m}$ is spread over this field. The source is placed at $\boldsymbol{\theta}^{*}=(5 \mathrm{~m}, 5 \mathrm{~m})$, with characteristics $\bar{P}_{0}=20 \mathrm{dBm}$, $d_{0}=1 \mathrm{~m}$. The measurement noise is such that $e=4 \mathrm{dBm}$. The path-loss exponent is $n_{\mathrm{p}}=2$, assumed constant over the field. The sampling time is $T=0.5 \mathrm{~s}$ and $[\mathbf{w}]=$ $[-0.5,0.5]^{2} \mathrm{~m} \cdot \mathrm{s}^{-2}$. Figure 2 illustrates the connectivity of the considered regular WSN and a typical trajectory followed by the source. Each sensor is connected in average with 6.9 sensors of the network.

The simplest algorithm implementation presented in Section 4.3 has been considered: sets are represented by boxes, simple image evaluations using inclusion functions are performed and correction is done by interval constraint propagation. This limits the amount of information to be exchanged between sensors and the computational effort. The localization performance using this algorithm is depicted in Figure 3 for 100 realizations of the source trajectory. The average width of the solution box (top of Figure 3) provided at each time instant decreases very quickly before reaching a floor slightly higher than the minimum width and increases again after about $18 \mathrm{~s}$. At the beginning, the source is close to the middle of the field and many sensors participate to the localization. When the sensor moves near the limits of the field, the number of involved sensors decreases and as a result the localization accuracy worsens. This effect is even more important when the source moves outside the field. A similar behavior is seen for the average norm of the localization error taking the center of the solution boxes at each time instant as estimate. The convergence is quite fast. The number of round trips impacts significantly the quality of the obtained results algorithm.
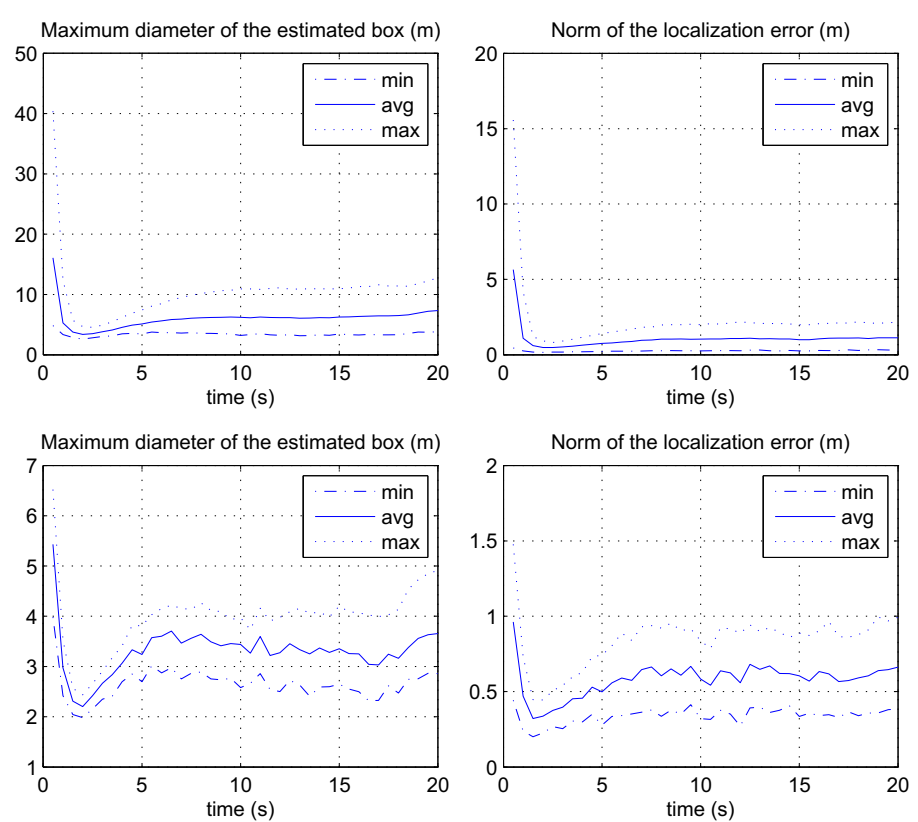

Fig. 3. Width of the box $\left[\theta_{1, k}\right] \times\left[\theta_{2, k}\right]$, and norm of the localization error when the estimate is taken as the center of the solution box, when one roundtrip (top) and three roundtrips (bottom) are allowed; results are averaged over 100 random paths followed by the source

\section{CONCLUSIONS}

In this paper, we have considered distributed boundederror state estimation applied to the problem of source tracking with a network of wireless sensors. Estimation is performed in a distributed context, i.e., each sensor has only a limited amount of measurements available. A guaranteed set estimator is put at work.

There is still large space for improvements in the considered problem. First, convergence properties have to be more carefully studied. In particular, more general conditions under which the distributed solution coincides with the centralized one have to be determined, in particular when wrappers are used instead of general sets. This type of problem is partly addressed in Yokoo [2001], Bejar et al. [2005]. Robustness to outliers has also to be considered. Extensions of Jaulin et al. [1996] have to be proposed in a distributed context.

\section{REFERENCES}

T. Alamo, J.M. Bravo, E.F. Camacho, and U. de Sevilla. Guaranteed state estimation by zonotopes. In Proceedings 42nd Conference on Decision and Control, 2003.

Peter Alriksson and Anders Rantzer. Experimental evaluation of a distributed Kalman filter algorithm. In Proceedings of the 46th IEEE Conference on Decision and Control, New Orleans, LA, December 2007. 
R. Bejar, C. Fernandez, M. Valls, C. Domshlak, C. Gomes, B. Selman, and B. Krishnamachari. Sensor networks and distributed CSP: Communication, computation and complexity. Artificial Intelligence Journal, 161(1-2): 117-148, 2005.

B. Bollobás. Modern Graph Theory. Springer-Verlag, NewYork, 1998.

H. Durrant-White, B. Rao, and H. Hu. Toward a fully decentralized architecture for multi-sensor data fusion. In Proc. IEEE International Conference on Robotics and Automation, volume 2, pages 1331-1336, 1990.

A. Gelb. Applied Optimal Estimation. MIT Press, Cambridge, MA, 1974.

S. Gezici, Z. Tian, G. B. Giannakis, H. Kobayashi, A. F. Molish, H. V. Poor, and Z. Sahinoglu. Localization via ultra-wideband radios. IEEE Signal Processing Magazine, 22(4):70-84, 2005.

F. Gustafsson and F. Gunnarsson. Mobile positioning using wireless networks. IEEE Signal Processing Magazine, 22(4):41-53, 2005.

T. Haenselmann. Sensornetworks. GFDL Wireless Sensor Network textbook. 2006. http://www.informatik.unimannheim.de/ $\tilde{h}$ aensel/sn_book.

F. Harary. Graph Theory. Addison-Wesley, Reading, MA, 1994.

A. O. Hero III and D. Blatt. Sensor network source localization via projection onto convex sets (POCS). In Proceedings of ICASSP, 2005.

L. Jaulin and E. Walter. Set inversion via interval analysis for nonlinear bounded-error estimation. Automatica, 29 (4):1053-1064, 1993.

L. Jaulin, E. Walter, and O. Didrit. Guaranteed robust nonlinear parameter bounding. In Proceedings of CESA'96 IMACS Multiconference (Symposium on Modelling, Analysis and Simulation), pages 1156-1161, Lille, France, 1996.

L. Jaulin, M. Kieffer, O. Didrit, and E. Walter. Applied Interval Analysis. Springer-Verlag, London, 2001.

R. E. Kalman. A new approach to linear filtering and prediction problems. Transactions of the AMSE, Part D, Journal of Basic Engineering, 82:35-45, 1960.

R. Kay and F. Mattern. The design space of wireless sensor networks. IEEE Wireless Communications, 11(6):54-61, 2004 .

M. Kieffer, L. Jaulin, and E. Walter. Guaranteed recursive nonlinear state estimation using interval analysis. In Proceedings of the 37th IEEE Conference on Decision and Control, pages 3966-3971, Tampa, FL, 1998.

M. Kieffer, L. Jaulin, I. Braems, and E. Walter. Guaranteed set computation with subpavings. In W. Kraemer and J. W. von Gudenberg, editors, Scientific Computing, Validated Numerics, Interval Methods, pages 167178, Boston, 2001.

M. Kieffer, L. Jaulin, and E. Walter. Guaranteed recursive nonlinear state bounding using interval analysis. International Journal of Adaptative Control and Signal Processing, 6(3):193-218, 2002.

P. Kontkanen, P. Myllymäki, T. Roos, H. Tirri, K. Valtonen, and H. Wettig. Probabilistic methods for location estimation in wireless networks. In R. Ganesh, S. Kota, K. Pahlavan, and R. AgustÍ, editors, Emerging Location Aware Broadband Wireless Adhoc Networks. Kluwer Academic Publishers, 2004.
J. A. López-Orozco, J. M. de la Cruz, E. Besada, and P. Ruiprez. An asynchronous, robust, and distributed multisensor fusion system for mobile robots. International Journal of Robotics Research, 19(10):914-932, 2000.

R. E. Moore. Interval Analysis. Prentice-Hall, Englewood Cliffs, NJ, 1966.

R. E. Moore. Methods and Applications of Interval Analysis. SIAM, Philadelphia, PA, 1979.

Y. Okumura, E. Ohmori, T. Kawano, and K. Fukuda. Field strength ans its variability in VHF and UHF landmobile radio service. Rev. Elec. Commun. Lab., 16:9-10, 1968.

R. Olfati-Saber. Distributed kalman filter with embedded consensus filters. In Decision and Control, 2005 and 2005 European Control Conference. CDC-ECC '05. 44th IEEE Conference on, pages 8179-8184, December 2005.

S. Piskorski, L. Lacassagne, M. Kieffer, and D. Etiemble. Efficient 16-bit floating-point interval processor for embedded systems and applications. In Proc. 12th GAMM - IMACS Int. Symp. on Scientific Computing, Computer Arithmetic and Validated Numerics (SCAN 2006), pages 23-23, 26-29 Sept. 2006. doi: 10.1109/SCAN.2006.15.

M. G. Rabbat and R. D. Nowak. Decentralized source localization and tracking. In Proc. ICASSP, 2004.

B. S. Y. Rao, H. F. Durrant-Whyte, and J. A. Sheen. A fully decentralized multi-sensor system for tracking and surveillance. International Journal of Robotics Research, 12(1):20-44, 1993.

A. Ribeiro, G. B. Giannakis, and S. I. Roumeliotis. SOIKF: Distributed Kalman filtering with low-cost communications using the sign of innovations. IEEE Trans. Signal Processing, 54(12):4782-4795, December 2006. ISSN 1053-587X. doi: 10.1109/TSP.2006.882059.

F. C. Schweppe. Uncertain Dynamic Systems. PrenticeHall, Englewood Cliffs, NJ, 1973.

X. Sheng and Y. H Hu. Maximum likelihood multiplesource localization using acoustic energy measurements with wireless sensor networks. IEEE Transactions on Signal Processing, 53(1):44 - 53, 2005.

B. Sklar. Digital Communications: Fundamentals and Applications. Prentice-Hall, 2001.

J. Speyer. Computation and transmission requirements for a decentralized linear-quadratic-gaussian control problem. IEEE Trans. Automatic Control, 24(2):266-269, April 1979. ISSN 0018-9286.

M. Yokoo. Distributed Constraint Satisfaction: Foundations of Cooperation in Multi-Agent Systems. SpringerVerlag, Berlin, 2001. 